\title{
Preprints of the
}

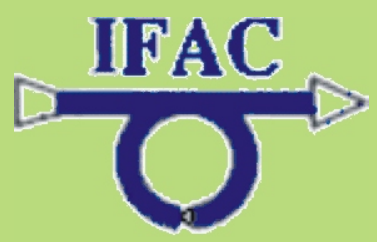

International Federation of Automatic Control

\section{2nd WORKSHOP ON LAGRANGIAN}

\section{AND HAMILTONIAN METHODS FOR NONLINEAR CONTROL}

\author{
April 3-5, 2003 \\ Seville, SPAIN
}

\section{Editors}

A. ASTOLFI, A.J. van der SCHAFT and F. GORDILLO 



\title{
DESCRIBING FUNCTION ANALYSIS OF MECHANICAL SYSTEMS WITH NONLINEAR FRICTION AND BACKLASH PHENOMENA
}

\author{
Ramiro S. Barbosa ${ }^{*}$, J. A. Tenreiro Machado*, Isabel M. Ferreira ${ }^{* *}$ \\ * Department of Electrotechnical Engineering \\ Institute of Engineering of Porto, Portugal \\ E-mail: \{rbarbosa,jtm\}@dee.isep.ipp.pt \\ ${ }^{* *}$ Department of Electrotechnical Engineering \\ Faculty of Engineering of Porto, Portugal \\ E-mail:imf@fe.up.pt
}

\begin{abstract}
This paper analyses the dynamical properties of systems with nonlinear friction and backlash phenomena based on the describing function method. The dynamics is illustrated using the Nyquist and Bode plots and the results are compared with those of standard models. Copyright (C) 2003 IFAC
\end{abstract}

Keywords: Describing function, Backlash, Friction, Mechanical Systems, Modelling.

\section{INTRODUCTION}

The phenomenon of vibration due to friction and impacts occurs in many branches of technology where it plays a very useful role. On the other hand, its occurrence is often undesirable, because it causes additional dynamic loads, as well as faulty operation of machines and devices. Despite many investigations that have been carried out so far, this phenomenon is not yet fully understood, mainly due to the considerable randomness and diversity of reasons underlying the energy dissipation involving the dynamic effects (Barbosa and Machado, 2002a, b).

In this paper we investigate the dynamics of systems that contain nonlinear friction and backlash with impacts. Bearing these ideas in mind, the article is organized as follows. Section 2 introduces the fundamental aspects of the describing function method. Sections 3 and 4 studies the describing function of mechanical systems with nonlinear friction and backlash phenomena, respectively. Finally, section 5 draws the main conclusions and addresses perspectives towards future developments.

\section{DESCRIBING FUNCTION ANALYSIS}

The describing function (DF) is one of the possible methods that can be adopted for the analysis of nonlinear systems (Slotine, and Li, 1991; Armstrong, and Amin, 1996; Armstrong, et al., 1994).
Let us consider the feedback control system of Fig. 1 with one nonlinear element $N$ and a linear system $G(s)$.

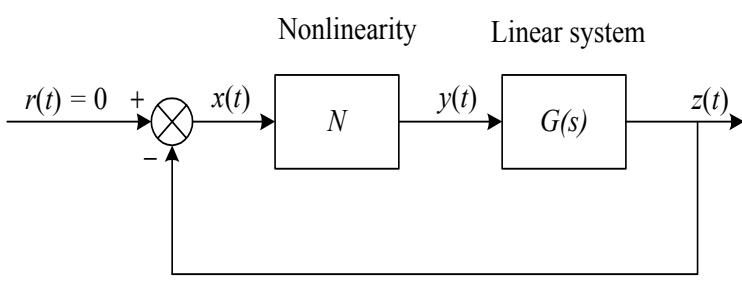

Fig. 1. Basic nonlinear feedback system for describing function analysis.

We start by applying a sinusoid $x(t)=X \sin (\omega t)$ to the nonlinearity input. At steady-state the output of the nonlinear characteristic, $y(t)$, is periodic and, in general, it is nonsinusoidal. If we assume that the nonlinearity is symmetric with respect to the variation around zero, the Fourier series becomes:

$$
y(t)=\sum_{k=1}^{\infty} Y_{k} \cos \left(k \omega t+\phi_{k}\right)
$$

where $Y_{k}$ and $\phi_{k}$ are the amplitude and the phase shift of the $k^{\text {th }}$-harmonic component of the output $y(t)$, respectively.

In the DF analysis, we assume that only $Y_{1}$, the fundamental harmonic component of $y(t)$, is significant. Such assumption is often valid since the 
higher-harmonics in $y(t), Y_{k}$ for $k=2,3, \ldots$, are usually of smaller amplitude than the amplitude of the fundamental component $Y_{1}$. Moreover, most systems are "low-pass filters" with the result that the higherharmonics are further attenuated.

Thus the DF of a nonlinear element, $N(X, \omega)$, is defined as the complex ratio of the fundamental harmonic component of output $y(t)$ with the input $x(t)$ :

$$
N(X, \omega)=\frac{Y_{1}}{X} e^{j \phi_{1}}
$$

where $X$ is the amplitude of the input sinusoid $x(t)$ and $Y_{1}$ and $\phi_{1}$ are the amplitude and the phase shift of the fundamental harmonic component of the output $y(t)$, respectively.

In general, $N(X, \omega)$ is a function of both the amplitude $X$ and the frequency $\omega$ of the input sinusoid. For nonlinear systems that do not involve energy storage, the DF is merely amplitude-dependent, that is $N=N(X)$. If it is not the case, we may have to adopt a numerical approach because, usually, it is impossible to find a closed-form solution.

For the nonlinear control system of Fig. 1, we have a limit cycle if the sinusoid at the nonlinearity input regenerates itself in the loop, that is:

$$
G(j \omega)=-\frac{1}{N(X, \omega)}
$$

Note that (3) can be viewed as the characteristic equation of the nonlinear feedback system of Fig. 1. If (3) can be satisfied for some value of $X$ and $\omega$, a limit cycle is predicted for the nonlinear system. Moreover, since (3) applies only if the nonlinear system is in a steady-state limit cycle, the DF analysis predicts only the presence or the absence of a limit cycle and cannot be applied to analysis for other types of time responses.

\section{SYSTEMS WITH NONLINEAR FRICTION}

This section analyses the DF of a dynamical system with nonlinear friction (Haessig, and Friedland, 1991; Armstrong, et al., 1994; Azenha, and Machado, 1998).

The system under consideration is a simple mass system with Coulomb plus Viscous plus Static friction (CVS model) as represented in Fig. 2.

The equation of motion in this system is as follows:

$$
M \ddot{x}(t)+F_{f}(t)=f(t)
$$

where $M$ is the system mass, $F_{f}(t)$ is the friction force and $f(t)$ the applied input force.

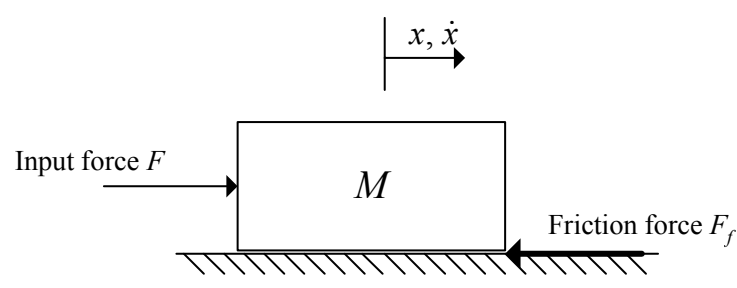

a)

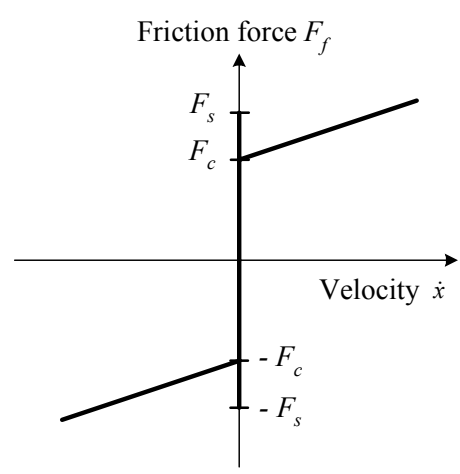

b)

Fig. 2. a) Elemental mass system subjected to nonlinear friction and b) Coulomb plus viscous plus static friction (CVS) model.

The discontinuity at zero velocity of the CVS model may lead to numerical problems in the simulations. To overcome the zero crossing detection and to get a reasonable numerical robustness we adopt the Karnopp model (Karnopp, 1985). The advantage of this model is that can produce sufficiently accurate results while reducing the algorithm complexity and simulation time. This model is described as:

$$
F_{f}(\dot{x}, F)=\left\{\begin{array}{cl}
F_{c} \operatorname{sgn}(\dot{x})+B \dot{x}, & |\dot{x}|>D_{v} \\
\min \left(|F|, F_{s}\right) \operatorname{sgn}(F), & |\dot{x}| \leq D_{v}
\end{array}\right.
$$

where $D_{v}$ specifies a small neighbourhood of the zero velocity (Karnopp, 1985). The parameters $F_{c}, F_{s}$ and $B$ are respectively the Coulomb, Static and Viscous friction level.

For the simple system of Fig. 2a we can calculate, numerically, the Nyquist plot of $-1 / \mathrm{N}(F, \omega)$ considering as input an sinusoidal force $f(t)=F \cos (\omega t)$ applied to mass $M$ and as output the position $x(t)$. Fig. 3 shows the function $-1 / \mathrm{N}(F, \omega)$ for several values of $F$.

The values of the parameters used in the simulations are: $M=1 \mathrm{Kg}, B=0.5 \mathrm{Ns} / \mathrm{m}, F_{c}=5 \mathrm{~N}$ and $F_{s}=7 \mathrm{~N}$. In the subsequent results the linear system case is also plotted for comparison purposes. 


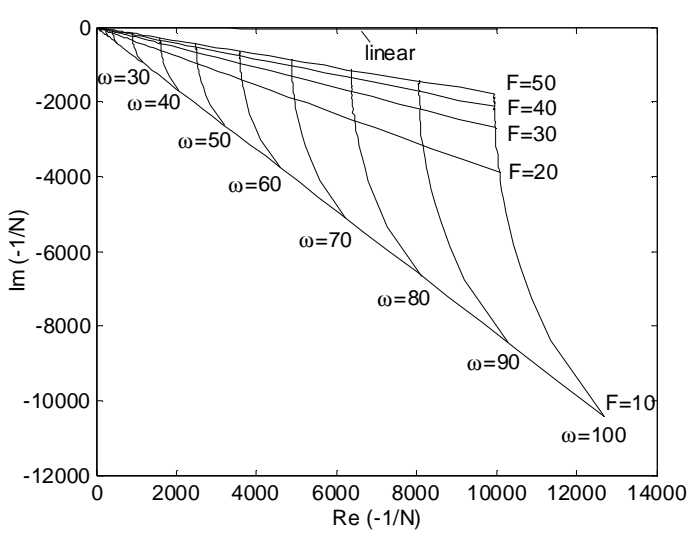

Fig. 3. Nyquist plot of $-1 / \mathrm{N}(F, \omega)$ for the system subjected to nonlinear friction and for an input force $F=\{10,20,30,40,50\}$ N.

Fig. 4 illustrates the variation of the Nyquist plots of $-1 / \mathrm{N}(F, \omega)$ for the cases of the linear system and nonlinear friction. The $\log -\log$ plots of $\operatorname{Re}\{-1 / \mathrm{N}\}$ and $\operatorname{Im}\{-1 / \mathrm{N}\}$ vs. the exciting frequency $\omega$, for different values of the input force $F=\{10,20,30,40,50\} \mathrm{N}$, reveal that we get results closer to the linear case the higher the excitation force $F$, particularly for the real component.
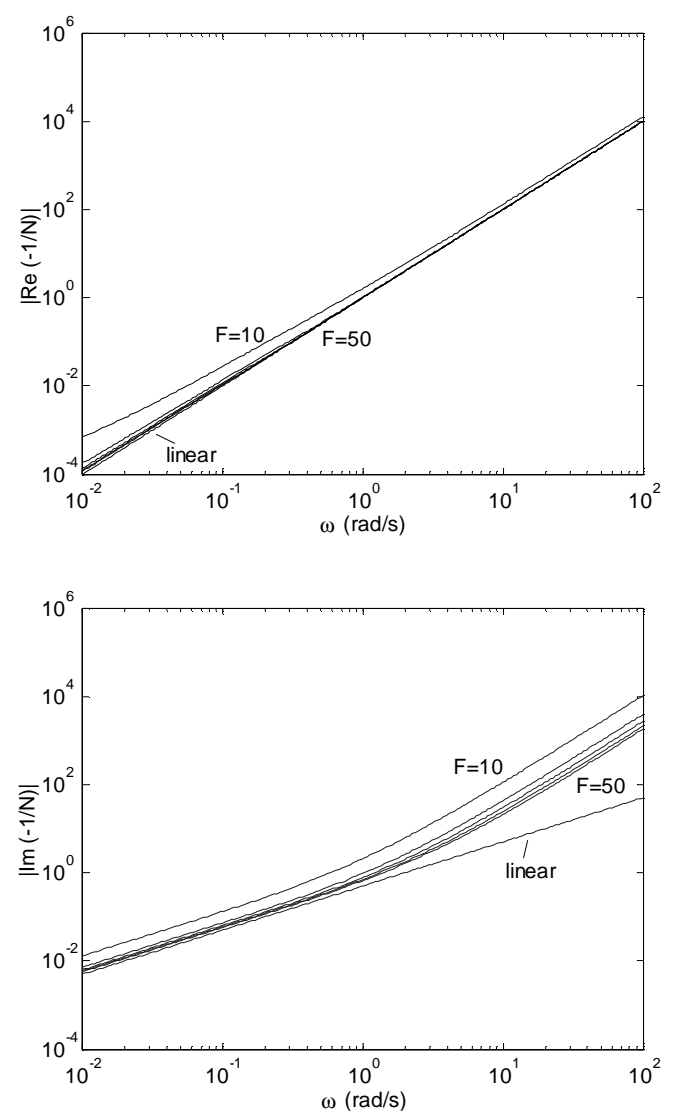

Fig. 4. $\log -\log$ plots of $\operatorname{Re}\{-1 / \mathrm{N}\}$ and $\operatorname{Im}\{-1 / \mathrm{N}\}$ vs. the exciting frequency $\omega$, for $F=\{10,20,30,40$, $50\} \mathrm{N}$.

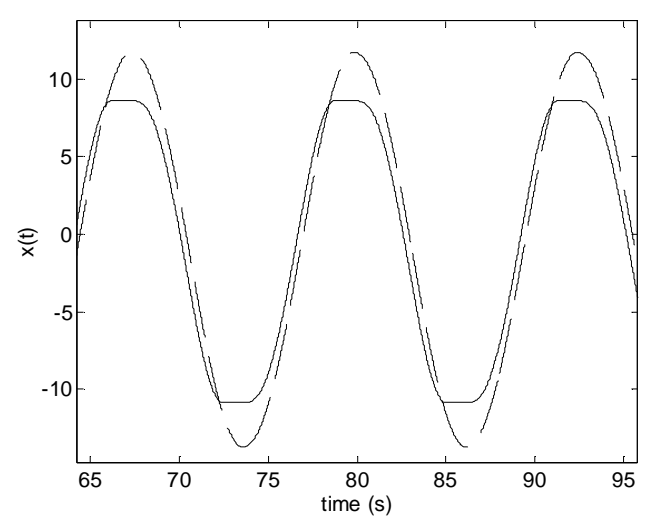

Fig. 5. Time response of the output position $x(t)$ of the system with nonlinear friction with $\omega=0.5$ $\mathrm{rad} / \mathrm{s}$, for $F=10 \mathrm{~N}$ (solid line) and $F=50 \mathrm{~N}$ (dashed line, scaled down by a factor of 10).

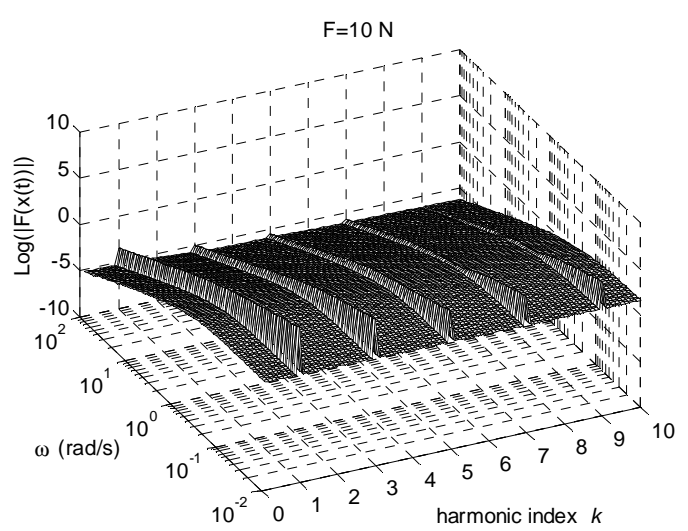

a)

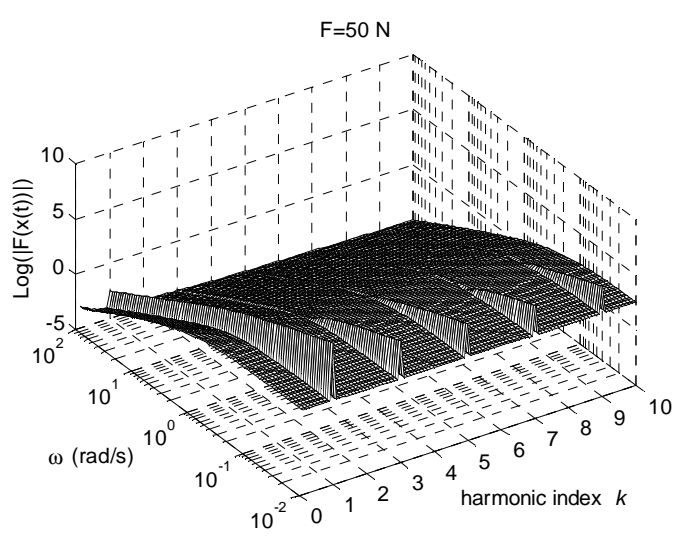

b)

Fig. 6. Fourier transform of the output position $x(t)$, $\mathrm{F}\{x(t)\}$, over 20 cycles, $v s .(\omega, k)$, the exciting frequency $\omega$ and the harmonic frequency index $k$ for: a) An input force $F=10 \mathrm{~N}$ and b) An input force $F=50 \mathrm{~N}$.

Fig. 5 compares the time response for an input force of $F=10 \mathrm{~N}$ and $F=50 \mathrm{~N}$. We conclude that for low forces the nonlinear effects of the static and Coulomb frictions are more significant, which is in accordance with the signal harmonic content $\mathrm{F}\{x(t)\}$ depicted in Fig.6. 


\section{SYSTEMS WITH BACKLASH AND IMPACTS}

In this section, we use the DF method to analyse the phenomena of clearance without and with the effect of the impacts, usually called static backlash and dynamic backlash, respectively (Barbosa, and Machado, 2002b; Nordin, and Gutman, 2002; Stepanenko, and Sankar, 1986; Dubowsky, et al., 1987; Choi, and Noah, 1989; Tao, and Kokotovic, 1995).

The standard approach to the backlash study is based on the adoption of a geometric model that neglects the dynamic phenomena involved during the impact process. Due to this reason often real results differ significantly from those predicted by that model.

The static backlash model leads to a DF of a linear system of a single mass $M_{1}+M_{2}$ followed by the geometric backlash having as input and as output the position variables $x(t)$ and $y(t)$, respectively, as depicted in Fig. 7 a.

The describing function for $X>h / 2$ is given by (Phillips and Harbour, 2000):

$$
\begin{gathered}
N(X)=\frac{k}{2}\left[1-N_{s}\left(\frac{X / h}{1-X / h}\right)\right]-j \frac{2 k h(X-h / 2)}{\pi X^{2}} \\
N_{s}(z)=\frac{2}{\pi}\left[\sin ^{-1} \frac{1}{z}+\frac{1}{z} \cos \left(\sin ^{-1} \frac{1}{z}\right)\right]
\end{gathered}
$$

For the dynamic backlash, the proposed mechanical model consists on two masses $\left(M_{1}\right.$ and $\left.M_{2}\right)$ subjected not only to backlash but also to impact phenomena as shown in Fig. $7 b$.

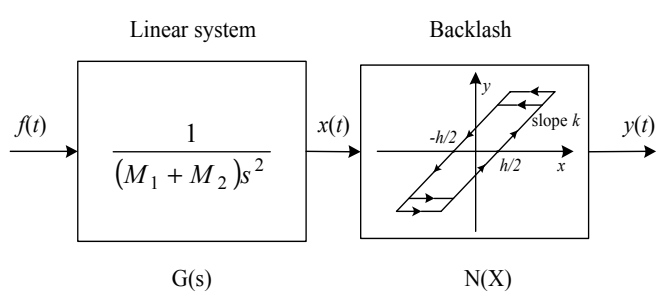

a)

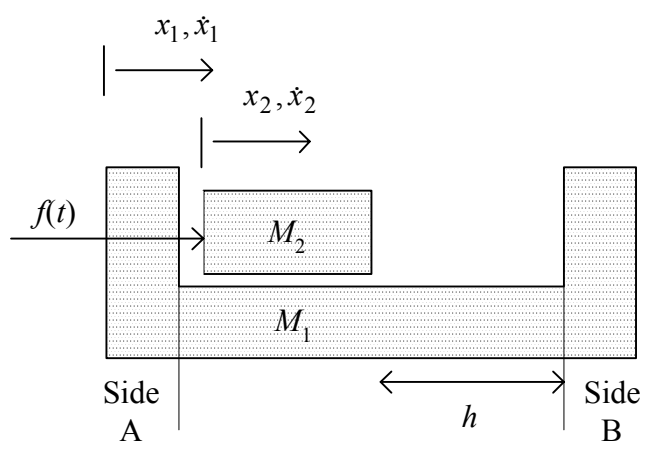

b)

Fig. 7. a) Classic backlash model and b) System with two masses subjected to dynamic backlash.
A collision between the masses $M_{1}$ and $M_{2}$ occurs when $x_{1}=x_{2}$ or $x_{2}=h+x_{1}$. In this case, we can compute the velocities of masses $M_{1}$ and $M_{2}$ after the impact ( $\dot{x}_{1}^{\prime}$ and $\dot{x}_{2}^{\prime}$ ) by relating them to the previous values ( $\dot{x}_{1}$ and $\left.\dot{x}_{2}\right)$ through Newton's law:

$$
\left(\dot{x}_{1}^{\prime}-\dot{x}_{2}^{\prime}\right)=-\varepsilon\left(\dot{x}_{1}-\dot{x}_{2}\right), \quad 0 \leq \varepsilon \leq 1
$$

where $\varepsilon$ is the coefficient of restitution. In the case of a fully plastic (inelastic) collision $\varepsilon=0$, while in the ideal elastic case $\varepsilon=1$.

By application of the principle of conservation of momentum $M_{1} \dot{x}_{1}^{\prime}+M_{2} \dot{x}_{2}^{\prime}=M_{1} \dot{x}_{1}+M_{2} \dot{x}_{2}$ and of expression (8), we can find the sought velocities of both masses after an impact:

$$
\begin{aligned}
& \dot{x}_{1}^{\prime}=\frac{\dot{x}_{1}\left(M_{1}-\varepsilon M_{2}\right)+\dot{x}_{2}(1+\varepsilon) M_{2}}{M_{1}+M_{2}} \\
& \dot{x}_{2}^{\prime}=\frac{\dot{x}_{1}(1+\varepsilon) M_{1}+\dot{x}_{2}\left(M_{2}-\varepsilon M_{1}\right)}{M_{1}+M_{2}}
\end{aligned}
$$

For the system of Fig. $7 \mathrm{~b}$ we calculate numerically the Nyquist diagram of $-1 / \mathrm{N}(F, \omega)$ for an input force $f(t)=F \cos (\omega t)$ applied to mass $M_{2}$ and considering the output position $x_{1}(t)$ of mass $M_{1}$.

The values of the parameters adopted in the subsequent simulations are: $M_{1}=M_{2}=1 \mathrm{Kg}$ and $h=10^{-1} \mathrm{~m}$. Figs. 8 and 9 show the Nyquist plots for $F=50 \mathrm{~N}$ and $\mathcal{\varepsilon}=\{0.1, \ldots, 0.9\}$ and for $F=\{10,20$, $30,40,50\} \mathrm{N}$ and $\varepsilon=\{0.2,0.8\}$, respectively.

The Nyquist charts of Figs. 8-9 reveal the occurrence of a jumping phenomenon, which is a characteristic of nonlinear systems. This phenomenon is more visible around $\varepsilon \approx 0.5$, while for the limiting cases $(\varepsilon \rightarrow 0$ and $\varepsilon \rightarrow 1)$ the singularity disappears. Moreover, Fig. 8 shows also that for a fixed value of $\varepsilon$ the charts are proportional to the input amplitude $F$.

The validity of the model is restricted to an input force $f(t)$ with frequency higher than a lower-limit $\omega_{C} \approx\left[\left(2 F / M_{2} h\right)^{2}(1-\varepsilon)^{5}\right]^{1 / 4}$ and lower than an upperlimit $\omega_{L}=2\left(F / M_{2} h\right)^{1 / 2}$, corresponding to an amplitude of $x_{1}(t)$ within the clearance $h / 2$. In the middle-range, $\omega_{C}<\omega<\omega_{\mathrm{L}}, \quad$ occurs a jumping phenomena at frequency $\omega_{J} \sim\left(F / M_{2} h\right)^{1 / 2}$.

Fig. 10 illustrates the variation of the Nyquist plots of $-1 / \mathrm{N}(F, \omega)$ for the cases of the static and dynamic backlash and shows the $\log -\log$ plots of $\operatorname{Re}\{-1 / \mathrm{N}\}$ and $\operatorname{Im}\{-1 / \mathrm{N}\}$ vs. $\omega$ for an input force $F=50 \mathrm{~N}$ and $\mathcal{E}=\{0.1,0.3,0.5,0.7,0.9\}$.

Comparing the results for the static and the dynamic backlash models we conclude that:

- The charts of $\operatorname{Re}\{-1 / \mathrm{N}\}$ are similar for low frequencies (where they reveal a slope of +40 
$\mathrm{dB} / \mathrm{dec}$ ) but differ significantly for high frequencies;

- The charts of $\operatorname{Im}\{-1 / \mathrm{N}\}$ are different in all range of frequencies. Moreover, for low frequencies, the dynamic backlash has a fractional slope inferior to $+80 \mathrm{~dB} / \mathrm{dec}$ of the static model.

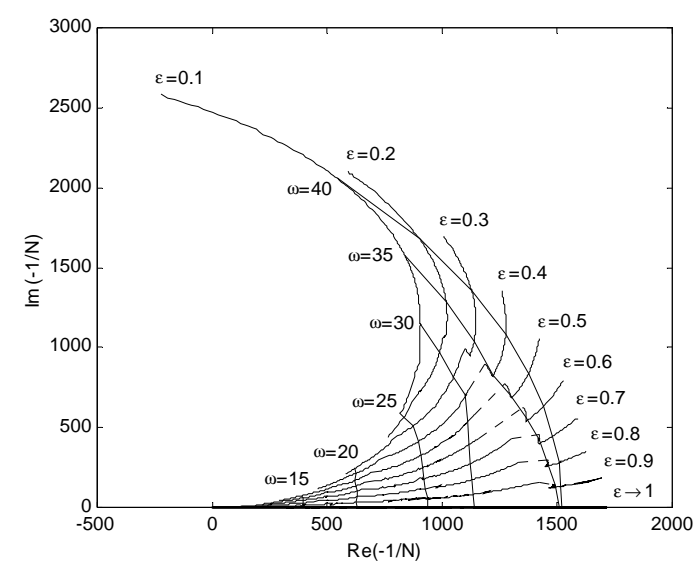

Fig. 8. Nyquist plot of $-1 / \mathrm{N}(F, \omega)$ for the dynamic backlash, $F=50 \mathrm{~N}$ and $\varepsilon=\{0.1, \ldots, 0.9\}$.
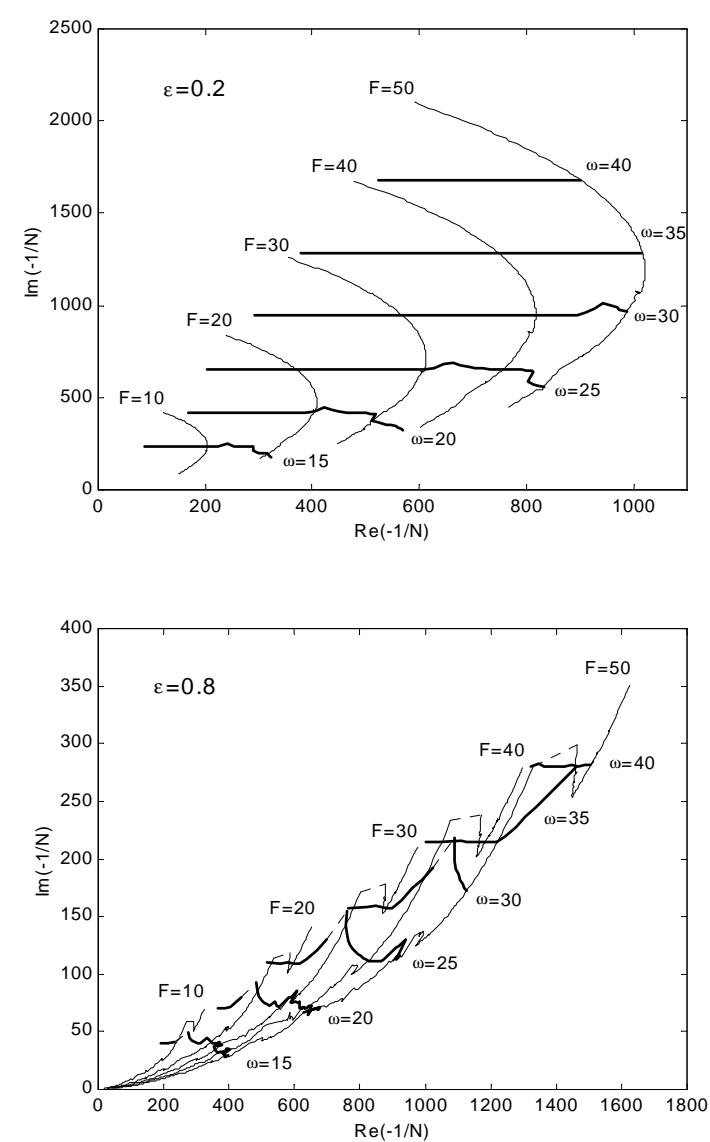

Fig. 9. Nyquist plots of $-1 / \mathrm{N}(F, \omega)$ for a system with dynamic backlash, $F=\{10,20,30,40,50\} \mathrm{N}$ and $\mathcal{E}=\{0.2,0.8\}$.
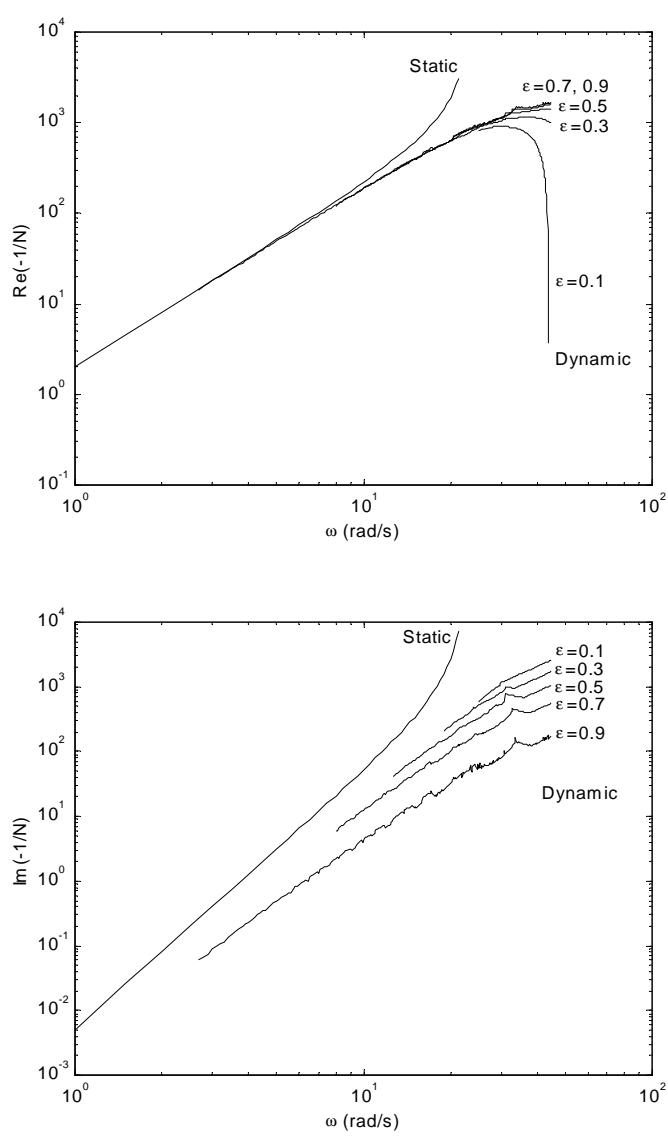

Fig. 10. $\log -\log$ plots of $\operatorname{Re}\{-1 / \mathrm{N}\}$ and $\operatorname{Im}\{-1 / \mathrm{N}\}$ vs. the exciting frequency $\omega$, for $F=50 \mathrm{~N}$ and $\mathcal{E}=\{0.1,0.3,0.5,0.7,0.9\}$.

Fig. 11 shows the time response of the output velocity $\dot{x}_{1}(t)$ of a system with dynamic backlash for $\omega=\{20,40\} \mathrm{rad} / \mathrm{s}$ and $\varepsilon=\{0.2,0.8\}$ revealing that we can have either chaotic or periodic responses depending on the values of $\omega$ and $\varepsilon$.

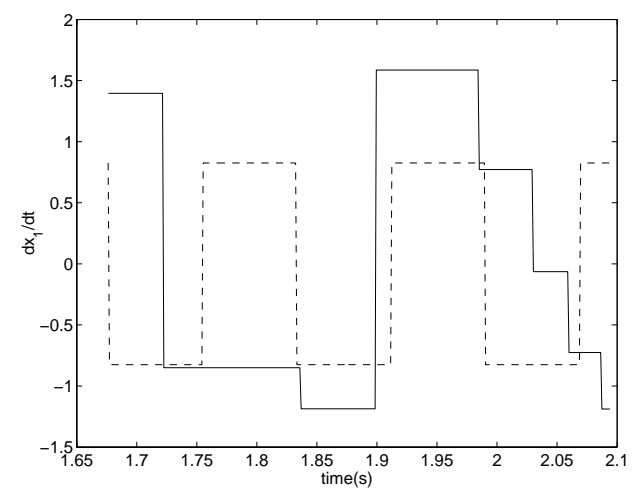

Fig. 11. Time response of the output velocity $\dot{x}_{1}(t)$ of the system with dynamic backlash, $F=50 \mathrm{~N}$ for $\omega=20 \mathrm{rad} / \mathrm{s}, \varepsilon=0.8$ (solid line) and $\omega=40 \mathrm{rad} / \mathrm{s}$, $\varepsilon=0.2$ (dotted line).

Fig. 12 presents the Fourier transform of the output displacement of mass $M_{1}, \mathrm{~F}\left\{x_{1}(t)\right\}$, namely the amplitude of the harmonic content of $x_{1}(t)$ for an input force $f(t)=50 \cos (\omega t), \omega_{C}<\omega<\omega_{L}$, and $\varepsilon=\{0.2,0.8\}$. The charts reveal that the fundamental harmonic of the 
output has a much higher magnitude than the other higher-harmonic components. This fact enables the application of the describing function in the prediction of limit cycles for this system. Nevertheless, for high values of $\varepsilon$, there is a significant high-order harmonic content, and by consequence, a lower precision of the limit cycle prediction.

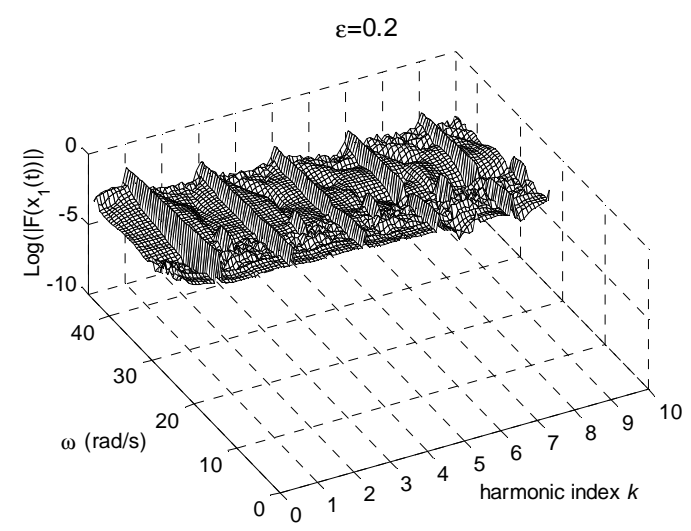

a)

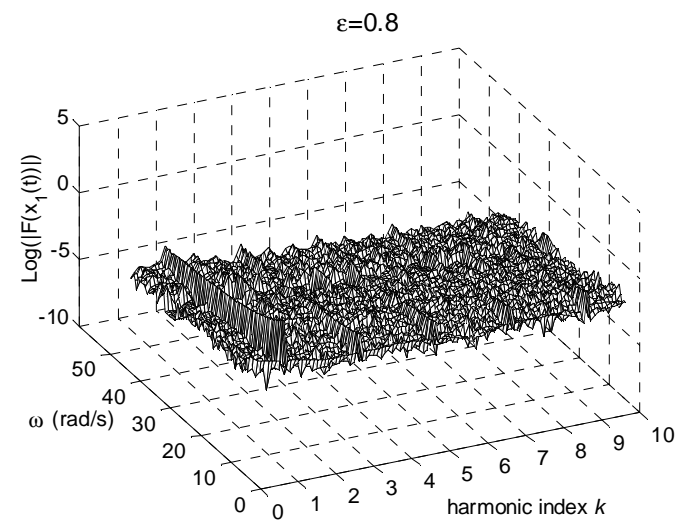

b)

Fig. 12. Fourier transform of the output displacement $x_{1}(t), \mathrm{F}\left\{x_{1}(t)\right\}$, over 20 cycles, vs. the exciting frequency $\omega$ and the harmonic index $k$ for: a) An restitution coefficient $\varepsilon=0.2$ and b) An restitution coefficient $\varepsilon=0.8$.

\section{CONCLUSIONS}

This paper addressed several aspects of the phenomena involved in systems with nonlinear friction and backlash. The dynamics of elemental mechanical system was analysed through the describing function method and compared with standard models. The results encourage further studies of nonlinear systems in a similar perspective and the analysis of limit cycle prediction. The conclusion may lead to the development of compensation schemes capable of improving control system performance.

\section{REFERENCES}

Armstrong-Hélouvry, B. and B. Amin (1996). PID Control in the Presence of Static Friction: A Comparison of Algebraic and Describing Function Analysis. Automatica, 32, 679-692.

Armstrong-Hélouvry, B., P. Dupont and C. Canudas de Wit (1994). A Survey of Models, Analysis Tools and Compensation Methods for the Control of Machines with Friction. Automatica, 30, 1083-1138.

Azenha, A. and J. A. T. Machado (1998). On the Describing Function Method and the Prediction of Limit Cycles in Nonlinear Dynamical Systems. SAMS Journal of Systems Analysis, Modelling and Simulation, 33, 307-320.

Barbosa, R. S. and J. T. Machado (2002a). Fractional Describing Function Analysis of Systems with Backlash and Impact Phenomena. In: Proc. of the $6^{\text {th }}$ Int. Conf. on Intelligent Engineering Systems 2002, pp. 521-526. Opatija, Croatia.

Barbosa, R. S. and J. T. Machado (2002b). Describing Function Analysis of Systems with Impacts and Backlash. Nonlinear Dynamics, 29, 235-250.

Choi, Y. S. and S. T. Noah (1989). Periodic Response of a Link Coupling with Clearance. ASME Journal of Dynamic Systems, Measurement and Control, 111, 253-259.

Dubowsky, S., J. F. Deck and H. Costello (1987). The Dynamic Modelling of Flexible Spatial Machine Systems with Clearance Connections. ASME Journal of Mechanisms, Transmissions and Automation in Design, 109, 87-94.

Haessig, D. A. Jr. and B. Friedland (1991). On the Modeling and Simulation of Friction. ASME Journal of Dynamic Systems, Measurement and Control, 113, 354-362.

Karnopp, D. (1985). Computer Simulation of StickSlip Friction in Mechanical Dynamic Systems. ASME Journal of Dynamic Systems, Measurement and Control, 107, 100-103 .

Nordin, M. and P. Gutman (2002). Controlling Mechanical Systems with Backlash-A Survey. Automatica, 38, 1633-1649.

Phillips, C. L. and R. D. Harbor (2000). Feedback Control Systems. Prentice-Hall, New Jersey.

Slotine, J. E. and W. Li (1991). Applied Nonlinear Control. Prentice-Hall, New Jersey.

Stepanenko, Y. and T. S. Sankar (1986). VibroImpact Analysis of Control Systems with Mechanical Clearance and its Aplication to Robotic Actuators. ASME Journal of Dynamic Systems, Measurement and Control, 108, 9-16.

Tao, G. and P. V. Kokotovic. Adaptive Control of Systems with Unknown Output Backlash (1995). IEEE Transactions on Automatic Control, 40, 326-330. 